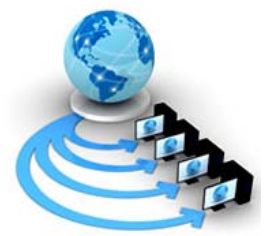

Volume 11, No. 4, July-August 2020

International Journal of Advanced Research in Computer Science

RESEARCH PAPER

\title{
NETWORK AIDED POSITIONING TECHNIQUES BASED ON RSSI AND SIGNAL PROCESSING
}

\author{
Putri Kevin \\ Department of Computer Science, \\ Brahijaya University, Indonesia
}

\begin{abstract}
The researcher's attentiveness has been diverted to create mobile positioning techniques based on RSSI and with signal processing techniques like GPS and multidimensional scaling. The localization system has been proposed in different topologies with a lot of tactical challenges in mobile positioning. Several positioning systems has been proposed for mobile based wireless sensor network and some are in a dense environment. In consequence, it is not practical to use a same localization system for different types of networks. Therefore, a system and algorithm are required which can calibrate itself and transform the global coordinates according to the environmental changes. In this article we provide an overview of different localization systems and provide some insight overview for future researchers.
\end{abstract}

Keywords: wireless sensor network, localization, RSSI, anchor node.

\section{INTRODUCTION}

The U.S Federal Communications commission precisely instructed to locate all E911 calls to facilitate emergency conditions. $95 \%$ of the earpieces sold by the end of December 2005 were location reconcilable. Other than enhanced 911 services wireless positioning has also been excessively helpful in many fields [1-2]. Diverse positioning systems have been offered for utilizing it in omnipresent computing. As an indispensable requirement for omnipresent computing linked with wireless networks, mobile users can ingress any kind of data with the liberty to share it anywhere [3]. A lot of other positioning systems has been presented in literature based on GPS, network aided techniques, mobile anchor-based localization and received signal strength localization. Global positioning system is a de facto standard for localization, but this system does not always work because sometime GPS might not available in indoor system, buildings and subways. GPS system also need line of sight and it may cost high because of the receiver cost [4].

These days, there are various Node Localization Algorithms with di erent precision and method. Regardless of the accuracy of certain calculations, this is as yet an opened research zone because of: [5] (I) processes like the compression, reflection, di raction, and dissipating. (ii) equipment requirements like capacity limit, the vitality utilization, the low computational power accessibility, and the radiation example of receiving wires; (iii) the sort of condition and (iv) Environment's components, like temperature, stickiness, and hurdles. The incorporation of each factor into a solitary node Localization Algorithm may impractical or practical to accomplish. A few answers for the limitation of nodes are run based NLA in which separations or points among hubs are utilized [6-7]. Some NLAs of this sort incorporate the Angle of Arrival, RSSI, ToA ,TDoA and GPS . GPS is not feasible, in light of the fact that the visible pathway is required and once in a while it isn't the best arrangement from a conservative point of view [8]. Then again, the range free based algorithms utilize data from obtained information. Some of the favourable aspects of such networks can be as follows [9]:

- Framework requirements: Due to smart sensor mapping at all nodes there is no settled framework which lowers the overhead expenditure and hence framework requirement.
- Mobility: These networks are less flexible in nature so can be applicable in applications such as video frames movement discovery [10].

- Multi-hop: Due to presence of multi nodes within a network a node can select multi-paths for one to one node communication which can save power linked with every node [11].

- Exposure: If position is unknown still data access is possible.

- Network dimension: Depends on number of nodes [12].

- Comparable network: Nodes present in a network has same characteristics.

- Location Knowledge: Every node should be familiar with its location with respect to other available nodes within a network and this is the main criteria for gathering information.

- Fault Lenient: With the particular node failure there would be no problem with the network process as same data may be available with any other node as well, but performance may degrade [13].

- Trustworthy information broadcast: For perfect requestresponse modelling node should demand to exact node and that node should respond fast and in an efficient manner for trustworthy processing.

- Data dependent: Data demanding is done as per the physical quantity.

- Addressing approach: The addresses have attribute-value pair where pair of value indicates which parameters are to be checked.

\section{LOCALIZATION AlgorithM’s}

Positioning systems can be categorized in different ways, considering indoor versus outdoor systems or cellular versus sensor network positioning. Here we can discuss the main branches of localization algorithms as well as their properties.

\section{A. Indoor versus outdoor systems:}

Even though GPS and Enhanced 911 services have labelled the question of location finding, but due to some technical 
issues they cannot provide precise geo-location indoor. Indoor geo-location utilizes the LAN foundation for spotting [14].

\section{B. Cellular versus sensor network positioning:}

Sensor networks range from traditional to autonomous networks. In sensor networks, location cognizance is essential. Sensor networks have a "Localization problem" in which it is necessary to know the site of sensor nodes. Sensor networks have broadly been used for environmental sensing, observing encroachment in battlefields, and observing wildlife [15-18].

\section{Network-aided positioning design:}

Various positioning system solutions are needed by distinct network topologies, media access control, and physical layer characteristics and social network analysis [19-21]. In this portion, we will be discussing the positioning solutions that are practical in wireless LANs, ad hoc sensors and cellular networks.

\section{Positioning in celluar networks:}

The nature of cellular network regulates whether a "standard" or "nonstandard" location technique should be used. This includes $2 \mathrm{G}$ and $3 \mathrm{G}$ networking and how they process [22-23].

\section{E. E-OTD for GSM:}

The most ordinary cellular grade in Europe is The Global System for Mobile Communication. The E-OTD locating method is deployed on the noted time variance (OTD) attribute of GSM systems. OTD records the time variance of signals moving from two distinct base transceiver stations (BTSs) to a mobile station (MS). These properties can affect the authenticity of E-OTD evaluation [24].

Location measurement unit (LMU) gadgets are hence needed to enumerate the clock distinctions between a base station and sending data to similar BTS's [22]. The BTSs then transmit the contemporize information to different mobile devices. So far, it is a costly solution. TDOAs [25] are incorporated to make bisecting hyperbolic lines that determine the location. E-OTD method proposes higher positioning authenticity than OTDs, having a steady retaliation of $5 \mathrm{~s}$. Furthermore, they need a software adapting receiver which signifies that they cannot be a helpful providing location.

\section{F. Assisted-GPS for Narrowband CDMA:}

Assisted GPS utilizes an earthbound cellular network to upgrade GPS receiver performance [26]. A GPS contains three parts; a location server, mobile station, and wireless communication connection. The mobile station and location server carry data to the GPS receiver. This data is called "assistance data", which is helpful for A-GPS. There are two categories of A-GPS; MS-assisted GPS and MS-based GPS.

\section{G. MS-Assisted-GPS:}

A single MS receives GPS satellite signals and regulates the pseudo ranges. These time-stamped pseudo ranges are delivered to the location server, which estimates the location, in comparison to GPS, A-GPS provides: 1) A higher rate of sensitivity for GPS receiver. 2) A depletion in the starting time to contemporize the GPS receiver. 3) An expanded positioning authenticity [27].

\section{H. MS-based-GPS:}

In this, MS can spot itself. The stumbling block is the design that cellular handsets should be provided with a disparate receiver [28]. Noise is also a main factor which can be reduced in several ways. Author in [29] have proposed a framework which efficiently remove the noise factor and increase the efficiency of coordinate systems. In another study fuzzy-logic approach also proven to be very much efficient in improving the overall localization accuracy [30].

\section{OTDoA for Wideband CDMA (WCDMA):}

As the name identifies, observed (OTDOA) is TDOArooted outlook. Just like E-OTD, OTDOA utilizes LMUs to divide into marked intervals, the measurements from neighboring base stations for a single mobile user. Taking into consideration the resemblance between the systems we can think of OTDOA as a WCDMA type of E-OTD. It has a similar deficiency as E-OTD [31]. The most notable provocation meet WCDMA location algorithms is an in-band intrusion. This impact is perceptible if a mobile station is near its serving area and needed to take calculation from neighboring base stations, a condition known as the "Near Far" complication. The OTDOA location method encounters issues such as posed by asynchronous base stations. The necessity to solve this issue has directed to the invention of idle period downlink (IPDL). The foundation behind the IPDL design is to incapacitate the work of base stations pseudo that will allow the mobile station to receive signals from neighboring tedious base stations during that short period.

\section{J. Cell-ID:}

It is a simple locating method based on cell sector data provided by the 3rd Generation Partnership Project (3GPP) [32]. Every cell is usually distributed into three sectors. The size of the cell differs from $1-3 \mathrm{~km}$ to $3-20 \mathrm{~km}$ in rural areas. The method's drawback is certain, location calculation authenticity relies on the cell sector strictly. To better the method's authenticity, Cell-ID+ TA (timing advance) and CellID + RTT (round trip time) hybrid positioning methods are suggested.

\section{NON-STANDARD LOCALIZATION ALGORITHM'S}

\section{A. Smart Antenna techniques for localization:}

Smart-Antenna-based location services utilize the angle of arrival (AOA) as a measuring variable. The system can instinctively modify the antenna's beam ornamentation, frequency retaliation, and other variables to intensify location performance. The location of MS is determined by two bisecting lines [33-36]. To fight inexactness more than two base stations might be engaged. A notable disadvantage of AOA is the need for specialized receivers at the base station. Another disadvantage is, an angular error can be translated to significant error. A strong amalgamation of GPS and CDMA can remarkably decrease hardware complexity. An additional TOA-AOA hybrid location spotting method relies on multilayer neural networks [37].

\section{B. Pattern matching for positioning:}

The solution to the question of AOA and TOA/TDOA difficulties is in the form of pattern matching for positioning which absorbs the "fingerprinting" of mobile phones. Whenever an E911 call is made location can be automatically found by signal values in the database. This resolves many issues of multipath propagation based on distance calculation [38-39]. 


\section{Positioning in Wireless LAN:}

WLANs are inaugurated by many customers to lower positioning price. The technical issues faced by WLANs are contrasting in frequency spectrums [40].

\section{Client based system design:}

Usually, the design uses two sections. First, the offline phase, the system is sectioned, and a model is made by receiving a limited number of positions in a specific area. Second, the online phase during which mobile units describe signal strength from the access point [41].

\section{E. Client-Assisted system design:}

In this system, client terminals, access points, and design work to place the client's location in WLAN. Time-stamp data is recorded. Sniffers might be placed with APs or at other positions [42].

\section{F. Positioning in AD-Hoc Sensor network:}

Determining the positions of nodes in ad hoc is quite difficult without using a usual clock. Besides, UWB methods are more reliable for indoor communication and geolocation. It can be divided into three divisions; Localization with beacons, Localization with moving beacons, and beacon-free localization [43].

\section{G. Beacon localization:}

In most networks, nodes are supplied with special positioning devices that locate them. Such nodes are called beacons. An unknown device could become a beacon by determining its location, so others can use its location services [44].

\section{H. Mobile beacon localization:}

In this network, nodes locate their positions by determining their distance from mobile observers. It indicates that the movement of targets can be utilized to remarkably enlarge the position estimation authenticity with a small number of reference nodes [45-46].

\section{Beacon-free localization:}

A very large network can be designed to work without beacons, which is known as beacon-free design. The conclusion has both translation and orientation degrees of freedom that have to measure accurately [47].

\section{ALGORITHM'S FOR AD-HOC NETWORKS}

\section{A. Beacon-based versus beacon-free algorithms:}

Beacon-based algorithms presume that a precise minimum number of nodes can determine their positions through GPS or manual configuration. In the first step, a heuristic is a recruit to develop a well spread, fold-free graph layout in resemblance to the desired layout. The second step utilizes a mass-spring model analogy.

\section{B. Incremental versus concurrent algorithms:}

Incremental algorithms start with only 3 or 4 core nodes being acknowledged of their coordinates. Some incremental algorithms can be practical for deceased stages of global optimization to decrease propagation error. They can also eliminate error propagation by decreasing global errors.

\section{Range-based vs range free:}

This kind of distance calculation is generally implemented utilizing signal strength decay, TOA, Or TDOA for internode range determination. Sensors spot themselves at the pivot of the location of beacons they detected. Utilizing hop-count as a suggestion of Euclidian distance, a sensor can determine its position with the help of triangulation. Another term, "rangefree" algorithm estimates whether a node lies inside or outside of the triangle.

\section{Single-hop versus multiple hop:}

In this system, nodes generally do not receive beacon nodes' signals. It is of no revelation that initial multi-hop localization algorithms tend to absorb single-hop technologies. It first estimated the gradient (hop distance) to the beacons (seeds) and determined the average hop distance to a beacon.

\section{CONCLUSION AND FUTURE WORK}

Wireless Localization has become significant. In this article, the art if the localization system and the use of algorithms have been proposed. Moreover, some notable issues have been highlighted that are still unsolved, and there is considerable want for coherent positioning, fault-forbearance, privacy, and security. Localization and positioning system still needed to concern for mobile based systems as well as BLE system where the design consideration and environmental factors are main concerns.

\section{REFERENCES}

[1] L. Gogolak, S. Pletl, and D. Kukolj, "Indoor fingerprint localization in WSN environment based on neural network," in Proceedings of the 9th IEEE International Symposium on Intelligent Systems and Informatics, pp. 293-296, Subotica, Serbia, September 2011.

[2] Nemer, I., Sheltami, T., Shakshuki, E., Elkhail, A. A., \& Adam. M. (2020). Performance evaluation of range-free localization algorithms for wireless sensor networks. PERSONAL AND UBIQUITOUS COMPUTING.

[3] Ahmad, T., Li, X. J., \& Seet, B. C. (2017). Parametric loop division for 3D localization in wireless sensor networks. Sensors, 17(7), 1697.

[4] Darade, Santosh Ashokrao, and M. Akkalakshmi. "Extensive Literature Survey on Load Balancing in Software-Defined Networking." International Journal of Business Data Communications and Networking (IJBDCN) 16, no. 2 (2020): 1-19.

[5] Sharma, Neha, and Vishal Gupta. "Meta-heuristic based optimization of WSNs Localisation Problem-a Survey." Procedia Computer Science 173 (2020): 36-45.

[6] Al-Salti, Faiza, N. Alzeidi, and Khaled Dav. "LOCALIZATION SCHEMES FOR UNDERWATER WIRELESS SENSOR NETWORKS: SURVEY."

[7] Dehkordi, Soroush Abbasian, Kamran Faraizadeh, Javad Rezazadeh, Reza Farahbakhsh, Kumbesan Sandrasegaran, and Masih Abbasian Dehkordi. "A survey on data aggregation techniques in IoT sensor networks." Wireless Networks 26, no. 2 (2020): 1243-1263.

[8] Ahmad, Tanveer, Xue Jun Li, and Boon-Chong Seet. "A self-calibrated centroid localization algorithm for indoor ZigBee WSNs." In 2016 8th IEEE International Conference on Communication Software and Networks (ICCSN), pp. 455-461. IEEE, 2016. 
[9] Dawadi, B. R., Rawat, D. B., Joshi, S. R., \& Keitsch, M. M. (2020). Towards energy efficiency and green network infrastructure deployment in Nepal using software defined IPv6 network paradigm. The Electronic Journal of Information Systems in Developing Countries, 86(1), e12114.

[10] Orozco, Felipe, Jing Li, Unwana Ezekiel, Zafarion Niyazov, Lauren Floyd, Guilherme MR Lima, Jozef GM Winkelman, Ignacio Moreno-Villoslada, Francesco Picchioni, and Raniita K. Bose. "Diels-Alder-based thermo-reversibly crosslinked polymers: Interplay of crosslinking density, network mobility, kinetics and stereoisomerism." European Polymer Journal (2020): 109882.

[11] Kim, Minyoung, and Jong-wook Jang. "A Study on Implementation of Multi-hop Network for LoRaWAN Communication." In 2020 International Conference on Information Networking (ICOIN), pp. 553-555. IEEE, 2020.

[12] Hu, S., Wang, G., Wang, Y.. Chen, C., \& Pan, Z. (2020). Accurate image super-resolution using dense connections and dimension reduction network. Multimedia Tools and Applications, 79(1-2), 1427-1443.

[13] Ravichandran, S. "Design and Development of Burden Lenient Network On-Chip Using FPGA."

[14] Ahmad, Tanveer, Xue Jun Li, and Boon-Chong Seet. "3D localization based on parametric loop division and subdivision surfaces for wireless sensor networks." In 2016 25th Wireless and Optical Communication Conference (WOCC), pp. 1-6. IEEE, 2016.

[15] Chai, M., Li, C., \& Huang, H. (2020). A New Indoor Positioning Algorithm of Cellular and Wi-Fi Networks. The Journal of Navigation, 73(3), 509-529.

[16] Khalife, Joe, Ceren Sevinc, and Zaher M. Kassas. "Performance Evaluation of TOA Positioning in Asynchronous Cellular Networks Using Stochastic Geometry Models." IEEE Wireless Communications Letters (2020).

[17] Feng, Pan, Danyang Oin. Guangchao Xu, Ruolin Guo, and Min Zhao. "Wireless sensor indoor positioning based on an improved particle filter algorithm." International Journal of Distributed Sensor Networks 16, no. 2 (2020): 1550147720903633.

[18] Varposhti, Marzieh, Vesal Hakami, and Mehdi Dehghan. "Distributed coverage in mobile sensor networks without location information." Autonomous Robots 44, no. 3 (2020): 627-645.

[19] Ahmad, Tanveer, Xue Jun Li, and Boon-Chong Seet. "3D localization using social network analysis for wireless sensor networks." In 2018 IEEE 3rd international conference on communication and information systems (ICCIS), pp. 88-92. IEEE, 2018.

[20] Pesce, V., Silvestrini, S., \& Lavagna, M. (2020). Radial basis function neural network aided adaptive extended Kalman filter for spacecraft relative navigation. Aerospace Science and Technology, 96, 105527.

[21] Forghani, M., Karimipour, F., \& Claramunt, C. (2020). From cellular positioning data to trajectories: Steps towards a more accurate mobility exploration. Transportation Research Part C: Emerging Technologies, 117, 102666.

[22] Khalife, Joe, Ceren Sevinç, and Zaher M. Kassas. "Performance Evaluation of TOA Positioning in Asvnchronous Cellular Networks Using Stochastic Geometry Models." IEEE Wireless Communications Letters (2020).
[23] Meer, Irshad A., Mustafa Ozger, and Cicek Cavdar. "On the Localization of Unmanned Aerial Vehicles with Cellular Networks." arXiv preprint arXiv:2003.04889 (2020).

[24] Rothschilds, Thomas, Remi Bernotavicius, Edward Brow, and William Ehlhardt. "Triggering the increased collection and distribution of monitoring information in a distributed processing system." U.S. Patent 10,678,671, issued June 9, 2020.

[25] Zou. Y.. \& Liu. H. (2020). TDOA Localization with Unknown Signal Propagation Speed and Sensor Position Errors. IEEE Communications Letters, 24(5), 1024-1027.

[26] Yuan, F., Jia, Z. Y., \& Cheng, E. (2020). Chirp-rate quasiorthogonality based DSSS-CDMA system for underwater acoustic channel. Applied Acoustics, 161, 107163.

[27] Wengler, M. J., Sheynblat, L., Moeglein, M. L., Biacs, Z. F.. \& Gum, A. J. (2020). U.S. Patent No. 10,534,088. Washington, DC: U.S. Patent and Trademark Office.

[28] Ning. W.. Jiang, P.. Guo, Y.. Wang, C., Tan, X., Zhang. W.. ... \& Xue, Y. (2020). GPS-Palm: a deep learningbased graphic presentation system for the prediction of Spalmitoylation sites in proteins. Briefings in Bioinformatics.

[29] Ahmad, Tanveer, Xue Jun Li, and Boon-Chong Seet. "Noise Reduction Scheme for Parametric Loop Division 3D Wireless Localization Algorithm Based on Extended Kalman Filtering." Journal of Sensor and Actuator Networks 8, no. 2 (2019): 24.

[30] Ahmad, Tanveer, Xue Jun Li, and Boon-Chong Seet. "Fuzzy-Logic Based Localization for Mobile Sensor Networks." In 2019 2nd International Conference on Communication, Computing and Digital systems (CCODE), pp. 43-47. IEEE, 2019.

[31] Grekhov, A., Kondratiuk, V., \& Ilnytska, S. (2020). RPAS communication channels based on WCDMA 3GPP standard. Aviation, 24(1), 42-49.

[32] Watfa, M., Wang, G., Ahmad, S., Adiakple, P. M., \& Olvera-Hernandez, U. (2020). U.S. Patent No. 10,581,813. Washington, DC: U.S. Patent and Trademark Office.

[33] Biswas, R. N., Mitra, S. K., \& Naskar, M. K. (2020). Energy Efficient and Secure Localization in Wireless Sensor Networks: An Approach Through Anchor Mobility Control. In Novel Advancements in Electrical Power Planning and Performance (pp. 250-282). IGI Global.

[34] Rehman, K., \& Nawaz, F. (2020). Localization of Static Passive RFID Tags with Mobile Reader in TwoDimensional Tag Matrix. Wireless Personal Communications, 1-20.

[35] Jayakrishnan, V. M., and Deepthy M. Vijayan. "Performance Analysis of Smart Antenna for Marine Communication." In 2020 2nd International Conference on Innovative Mechanisms for Industry Applications (ICIMIA), pp. 88-91. IEEE, 2020.

[36] Pala, Sreenivasulu, Srividhya Palliyani, Mohamed Himdi, Olivier Lafond, and Dhanesh G. Kurup. "Localization of unknown electromagnetic source using 3D-antenna arrays." International Journal of Microwave and Wireless Technologies 12, no. 1 (2020): 86-94.

[37] Maletic, Neboisa, Vladica Sark, Marcus Ehrig, Jesus Gutierrez, and Eckhard Grass. "Performance Evaluation of LoS Round-Trip ToF Localization: A $60 \mathrm{GHz}$ Band Case Study." In WSA 2020; 24th International ITG Workshop on Smart Antennas, pp. 1-6. VDE, 2020.

[38] Ahmad, T. (2019). 3D Localization Techniques for Wireless Sensor Networks (Doctoral dissertation, Auckland University of Technology). 
[39] Meng, J., Ren, M., Wang, P., Zhang, J., \& Mou, Y. (2020). Improving Positioning Accuracy via Map Matching Algorithm for Visual-Inertial Odometer. Sensors, 20(2), 552.

[40] Lee, Donghun, Kyungin Min, and Jungha Kim. "Wireless LAN-based Vehicle Location Estimation in GPS Shading Environment." The Journal of The Korea Institute of Intelligent Transport Systems 19, no. 1 (2020): 94-106.

[41] Khan. Akif. Shah Khusro, Badam Niazi, Jamil Ahmad, Iftikhar Alam, and Inavat Khan. "TetraMail: a usable email client for blind people." Universal Access in the Information Society 19, no. 1 (2020): 113-132.

[42] Zhang, W., Sharma, A., \& Wood, T. (2020). EdgeBalance: Model-Based Load Balancing for Network Edge Data Planes. In 3rd \{USENIX\} Workshop on Hot Topics in Edge Computing (HotEdge 20).

[43] Sakr, M., Masiero, A., \& El-Sheimy, N. (2020). LocSpeck: A Collaborative and Distributed Positioning
System for Asymmetric Nodes Based on UWB Ad-Hoc Network and Wi-Fi Fingerprinting. Sensors, 20(1), 78.

[44] Oh, Hyun Oh, Juhyung Son, Jin Sam Kwak, and K. O. Geoniung. "System and method for indoor localization using beacons." U.S. Patent 10,571,547, issued February 25, 2020.

[45] Guo, Ying, Qinghe Han, and Xiaoyue Kang. "Underwater sensor networks localization based on mobilityconstrained beacon." Wireless Networks 26, no. 4 (2020): 2585-2594.

[46] Ahmad, Tanveer, Xue Jun Li, Boon-Chong Seet, and Juan-Carlos Cano. "Social Network Analysis Based Localization Technique with Clustered Closeness Centrality for 3D Wireless Sensor Networks." Electronics 9, no. 5 (2020): 738.

[47] hakila, R., and B. Paramasivan. "An improved range based localization using Whale Optimization Algorithm in underwater wireless sensor network." Journal of Ambient Intelligence and Humanized Computing (2020): 1-11. 